\title{
Gene-nutrient interactions in the metabolic syndrome: the role of adiponectin
}

\author{
J. F. Ferguson ${ }^{1}$, C. Phillips ${ }^{1}$, A. C. Tierney ${ }^{1}$, J. McMonagle ${ }^{1}$, J. A. Lovegrove ${ }^{2}$, H. Lovdal Gulseth ${ }^{3}$, C. \\ Defoort $^{4}$, E. Blaak ${ }^{5}$, C. Marin ${ }^{6}$, L. Partyka ${ }^{7}$, B. Karsltröm ${ }^{8}$, B. Vessby ${ }^{8}$, A. Dembinska-Kiec ${ }^{7}$, J. López \\ Miranda $^{6}$, W. Saris ${ }^{5}$, D. Lairon ${ }^{4}$, C. A. Drevon ${ }^{3}$, C. M. Williams ${ }^{2}$ and H. M. Roche \\ ${ }^{1}$ Nutrigenomics Research Group, Conway Institute, University College Dublin, Dublin, Republic of Ireland, ${ }^{2}$ Hugh Sinclair \\ Unit of Human Nutrition, School of Food Biosciences, University of Reading, Reading, UK, ${ }^{3}$ Department of Nutrition, \\ Institute of Basic Medical Sciences, University of Oslo, Oslo, Norway, ${ }^{4}$ INSERM 476, Nutrition Humaine et lipids, \\ INRA 1260, Université de la Méditerranée Aix-Marseille 2, Marseille, France, ${ }^{5}$ Maastricht University, Maastricht, \\ The Netherlands, ${ }^{6}$ Lipid and Atherosclerosis Unit, School of Medicine, University of Cordoba, Cordoba, Spain, \\ ${ }^{7}$ Department of Clinical Biochemistry, Jagiellonian University Medical College, Krakow, Poland and ${ }^{8}$ Department of \\ Public Health \& Caring Sciences/Clinical Nutrition and Metabolism, Uppsala University, Uppsala, Sweden
}

The metabolic syndrome (MetS) is a complex disorder characterised by abdominal obesity, insulin resistance, hypertension, dyslipidaemia and inflammation ${ }^{(1)}$. Development of the MetS is thought to be influenced by both genetic and environmental effects, with diet being a major environmental factor. Multiple genes are involved in the disease and interactions between gene variants and dietary fatty acids may modulate the risk of the disease.

The LIPGENE WP1.2 project (Diet, genomics and the metabolic syndrome: an integrated nutrition, agro-food, social and economic analysis) aims to investigate associations between polymorphisms in candidate genes and gene-nutrient interactions with biomarkers of the MetS, by means of a dietary intervention to alter fat composition in a cohort of patients with MetS.

The adiponectin gene (ACDC, ADIPOQ) codes for the adiponectin protein, the most abundant adipokine secreted by adipocytes ${ }^{(2)}$. Levels of circulating adiponectin are reduced in obese subjects and subjects with type 2 diabetes, as well as in patients suffering from a variety of obesity-related malignancies. This hormone may be a key player in the aetiology of the MetS, being an important regulator of insulin sensitivity and inflammation. An investigation has been conducted into whether polymorphisms in ACDC are associated with biomarkers of the MetS, and whether polymorphisms in other genes are associated with plasma levels of adiponectin, taking into account both dietary fat intake and plasma fatty acid levels in 486 individuals at baseline.

In total four single-nucleotide polymorphisms (SNP) within the adiponectin gene (ACDC: rs266729) and its 2 receptors (ADIPOR1: rs10753929, rs10920533 and ADIPOR2: rs6489323) showed significant associations $(P \leq 0.006)$ with fasting insulin levels when levels of plasma SFA were taken into account. In general, higher plasma SFA was accompanied by higher fasting insulin; however, this association was only demonstrated in a subset of the population, with the effect being dependent on genotype. Thus, some genotypes appear to interact with plasma SFA to exert a modifiable effect on fasting insulin, while others are unresponsive to SFA levels. A combination of the four SNP with plasma SFA demonstrated an even greater significant effect $\left(P=0.001, \eta^{2} 0.186\right)$. Levels of circulating adiponectin were also found to be associated with particular combinations of genotypes and SFA. Individuals showed significant tendencies $(P \leq 0.02)$ towards either stable or varying levels of adiponectin in response to differences in plasma SFA levels, depending on genotype in the following SNP: rs2241766 in ACDC, rs10920533 in ADIPOR1 and rs1800783 in NO synthase 3. Combination of these three SNP further increased the effect $\left(P=0.000, \eta^{2} 0.11\right)$. Using information from single SNP or SNP combinations could thus potentially identify individuals who are more likely to respond to dietary therapy.

These results provide further evidence towards the emerging role of adiponectin in obesity and related disorders, and provide optimism for the use of nutrigenomic approaches in disease management. While alterations in fat composition may not be sufficient to improve symptoms in all patients, the results demonstrate that certain genotypes may give rise to more modifiable phenotypes that are more sensitive to levels of SFA. These individuals might respond more positively to dietary interventions, reducing the need for drug treatment, or slowing disease progression.

This work was supported by the European Commission, Framework Programme 6 (LIPGENE): contract number FOOD-CT-2003-505944. The LIPGENE WP1.2 project is an EU Sixth Framework Programme Integrated Project.

1. Moller DE, Kaufman KD. Metabolic syndrome: A clinical and metabolic perspective. Annu Rev Med 2004; 56, 45-62.

2. Vasseur F, Meyre D, Froguel P. Adiponectin, type 2 diabetes and the metabolic syndrome: lessons from human genetic studies. Expert Rev Mol Med 2006 Nov $20 ; 8(27), 1-12$. 